\title{
Does oval oocyte have an impact on embryo development in in vitro fertilization?
}

\author{
Binarwan Halim ${ }^{1}$, Hilma Putri Lubis ${ }^{1}$, Diana Novia ${ }^{1}$, Masithah Thaharuddin ${ }^{1}$
}

${ }^{1} \mathrm{HFC}$ IVF center Division of Reproductive, Endocrinology and Infertility Department of Obstetrics and Gynecology, Faculty of Medicine University of Sumatera Utara, Medan, Indonesia.

\begin{abstract}
Objective: To compare the outcomes of embryo development between oval-shaped oocyte as an abnormal morphology oocyte and a normal morphology oocyte in In Vitro Fertilization (IVF).

Methods: This study was a comparative analytical study with retrospective approach which lasted from September 2014 until November 2015. For this study, we used secondary data (medical records) from 24 patients submitted to IVF at the Halim Fertility Center. The oocyte morphology was divided into two groups: normal-shaped oocyte and oval-shaped oocyte.

Results: Our study included 120 oocytes with 60 oval-shaped oocytes and 60 normal oocytes. We found fertilization rates of $68.3 \%$ in the normal oocyte group and $61.7 \%$ in the oval-shaped oocyte group; and there was no significant difference between the normal oocyte group and the oval-shaped oocyte group $(p>0.05)$. In the normal oocyte group, $65 \%$ had reached day 3 embryos, and in the oval-shaped oocyte group it was $50 \%$, with no significant difference between the groups $(p>0.05)$. We also found $46.7 \%$ transferrable embryos in the oval oocytes group compared to $63.3 \%$ in the normal oocytes group, with no significant difference between the groups $(p>0.05)$.

Conclusion: There was no significant difference in fertilization rates and embryo quality between normal morphology oocytes and oval-shaped oocytes.
\end{abstract}

Keywords: In vitro fertilization, normal oocyte, oval oocyte, dimorphism, morphology

\section{INTRODUCTION}

Human reproduction is the result of a union between two highly specialized cells- the oocyte and the spermatozoon. Of these, the oocyte deserves special mention because of its key functions; it receives the spermatozoon during fertilization, contributes much of the cytoplasm for early embryo development and provides half of the genome for one the resulting zygotes (Savitha et al., 2012; Plachot, 2000).

The correlation between oocyte quality, embryo development, and in-vitro fertilization (IVF) outcomes has been widely studied (Lazzaroni-Tealdi et al., 2015). Some studies revealed that successful pregnancy outcome with IVF/intracytoplasmic sperm injection (ICSI) depended on several variables including oocyte and sperm quality (Savitha et al., 2012; Braga et al., 2013; Ebner et al., 2006).

Oocyte quality has been regarded as a variable that may influence embryo quality (Plachot, 2000). Oocyte and embryo quality were assessed based on morphological features (Lazzaroni-Tealdi et al., 2015; Braga et al., 2013; ALPHA Scientists In Reproductive Medicine \& ESHRE Special Interest Group Embryology, 2011). However, the correlation between these "abnormal" oocyte morphotypes, fertilization rates, and embryo development is still unclear. The most frequently observed oocyte morphologic variations are cytoplasmic changes, including granularity, darkness, vacuole presence. Extracytoplasmic variations are oocyte shape, zona pellucida (ZP), perivitelline space (PVS), and polar body morphology (Savitha et al., 2012; Lazzaroni-Tealdi et al., 2015; Braga et al., 2013). Oocyte dimorphism such as oval (ovoid) oocyte or other abnormal oocyte morphology may affect fertilization rate and embryo development (Ebner et al., 2000; 2008; Xia, 1997; Serhal et al., 1997). Morphological Oocyte abnormalities also correlated to aneuploidy embryo in IVF cycle (Dolgushina et al., 2015). The aim of this study was to compare outcomes of embryo development between oval-shaped oocytes and normal morphology oocytes in IVF.

\section{MATERIAL AND METHODS}

This study was a comparative analytical study with a retrospective approach, which ran from September 2014 until November 2015. The subject of the study was secondary data (medical records) recruited from 24 patients who underwent IVF procedure at the Halim Fertility Center, Division of Reproductive Endocrinology and Infertility, Department of Obstetrics \& Gynecology, Faculty of Medicine - University of Sumatera Utara, Haji Adam Malik General Hospital, Medan, Indonesia. Oocyte and embryo quality were assessed based on morphological features. Oocyte morphology was divided into two groups: normal morphology oocyte and oval-shaped oocyte. Samples were selected consecutively to reach the minimum samples requirement based on the inclusion and exclusion criteria. The Inclusion criteria were all woman who underwent IVF procedure with PCOS, tubal factor, endometriosis, idiopathic infertility and male factor. The exclusion criterion was incomplete medical records.

This study was approved by the ethics committee of the faculty of Medicine of the University of Sumatera Utara.

\section{Controlled Ovarian Hyperstimulation}

The standard protocol for controlled ovarian stimulation was the short protocol, carried out using recombinant follicle-stimulating hormone (FSH) (Gonal-F; Serono), 150300 IU as a daily dose, starting on day 2 of the cycle. We used a gonadotropin-releasing hormone ( $\mathrm{GnRH})$ antagonist (Cetrotide; Serono), at a dose of $0,25 \mathrm{mg}$ to prevent a premature luteinizing hormone (LH) surge, starting when at least one follicle $>14 \mathrm{~mm}$ was visualized. Follicular growth was monitored using transvaginal ultrasound, starting on day 4 of the gonadotropin administration. The average of stimulation duration was 10-12 days. Oocyte maturation was triggered with recombinant hCG (Ovidrel; Serono), when the lead follicles reached $17-18 \mathrm{~mm}$. Oocytes were retrieved 36 hours after the injection of hCG by transvaginal ultrasound guiding the needle aspiration of follicles. 


\section{Oocyte Morphology Assessment}

Retrieved oocytes were maintained in culture medium supplemented (G-IVF Plus, Vitrolife) with $10 \%$ protein supplement and covered with paraffin oil for 2 to 3 hours before removing cumulus cells. The surrounding cumulus cells were removed after exposure to an N-2-hydroxyethylpiperazineN0-2-ethanesulfonic acid (HEPES)-buffered medium containing hyaluronidase (SynVitro ${ }^{\mathrm{TM}}$ Hyadase, Origio). The remaining cumulus cells were mechanically removed by gently pipetting with a hand-drawn Pasteur pipette. Oocyte morphology was assessed just before sperm injection (4 hours after retrieval) using an inverted Nikon Diaphot (stereomicroscope Nikon smz 1000+) microscope. The oocytes were classified as normal and abnormal (oval) according to their morphologic appearance.

\section{ICSI Procedure}

The Intracytoplasmic Sperm Injection was performed in a microinjection dish prepared with $10-\mu \mathrm{L}$ droplets of buffered medium (G-MOPS Plus, Vitrolife) and covered with paraffin oil on the heated stage of an inverted microscope $\left(37 \pm 0.5^{\circ} \mathrm{C}\right.$ ) (Nikon Eclipse TE2000). Approximately 17 hours after ICSI, fertilization was confirmed by the presence of two pronuclei and the extrusion of the second PB. The embryo was cultured at $37^{\circ} \mathrm{C}$ with the level of $\mathrm{CO}_{2}$ at $6 \%$ and $\mathrm{O}_{2}$ at $5 \%$. We used ISM1 medium (MediCult) and cultured until day 3 (approximately 68 hours), then we performed fresh or freezing embryo transfer.

\section{Embryo Morphology Assessment}

Embryo morphology was assessed at the zygote stage (16-18 hours after ICSI) and on the mornings of days 2 and 3 of embryo development, using an inverted Nikon Diaphot microscope (stereomicroscope Nikon smz 1000+) with a Hoffmann modulation contrast system under x200 magnification. Immediately before ET we assessed the embryo morphology.

To evaluate the cleavage-stage morphology, the following parameters were recorded based on the Istanbul consensus workshop on embryo assessment (ALPHA Scientists In Reproductive Medicine \& ESHRE Special Interest Group Embryology, 2011): the number of blastomeres, the percentage of fragmentation, the variation in blastomere symmetry, the presence of multinucleation, and defects in the ZP and cytoplasm. The high-quality cleavage stage embryos were defined as those with all the following characteristics: 4 cells on day 2 or 8-10 cells on day $3,<10 \%$ fragmentation, symmetric blastomeres, the absence of multinucleation, colorless cytoplasm with moderate granulation and no inclusions, the absence of PVS granularity, and the absence of ZP dimorphism. Embryos lacking any of these characteristics were of low quality (Table 1 ).

\section{Data Analysis}

We tabulated data on oocyte morphology and embryo development. Embryo development outcomes were determined by fertilization rates, cleavage rates on day 3 and transferrable embryo. Data was analyzed by computer applications in accordance with comparative analytical data presentations. To analyze embryo development outcomes between normal morphology oocyte and oval morphology oocyte, we used the chi-square statistical test with a $95 \%$ confidence interval. A P value of $<0.05$ was considered statistically significant.

\section{Sample size Calculation}

In this study, we used a comparative analytical study with retrospective approach. Therefore, this was the sample size calculation.

$$
n_{1}=n_{2}=\frac{\left(Z \alpha \sqrt{2 P Q}+Z \beta{\sqrt{P_{1} Q_{1}+P_{2} Q_{2}}}^{2}\right)}{\left(P_{1}-P\right)_{2}^{2}}
$$

$\mathrm{Za}=$ normal default value of $\mathrm{Z}$ table that depends on the value of $a$ is determined. $a$ value $=0.05 \rightarrow Z a=1.96$

$Z \beta$. = normal default value of table $Z$ that depends on the value of $\beta$ is determined. $\beta$ value $=0,20 \rightarrow Z \beta=0.84$

$\mathrm{P} 1=$ the proportion of fertilized oocytes with normal morphology $=0.9$

$\mathrm{P} 2=$ the proportion of fertilized oocytes with oval shape $=0.6$

$\mathrm{Q} 1=1-\mathrm{P} 1=0.1$

$\mathrm{Q}=1-\mathrm{P}=0.2$

$\mathrm{Q} 2=1-\mathrm{P} 2=0.3$

$\mathrm{P}=(\mathrm{P} 1+\mathrm{P} 2) / 2=0.8$

$\mathrm{n} 1=\mathrm{n} 2=$ total sample $=32$ oocytes (minimum total sample)

Therefore, the total sample in this study was 60 oocytes (each sample)

\section{RESULTS}

From September 2014 until November 2015, we included 120 oocytes recruited from 24 patients, being 60 with oval shape and 60 normal morphology oocytes.

From table 2, the fertilization rate in the normal morphology oocyte group was $68,3 \%$ in 41 oocytes and the fertilization rate in the oval-shaped oocyte group was $61.7 \%$ in 37 oocytes. The Statistical analysis showed no significant difference in fertilization rates between the normal oocyte group and the oval oocyte group $(p=0.444)$.

This table also showed that the cleavage outcome at day 3 in the normal oocyte group was $65 \%$ in 39 oocytes and in the oval-shaped oocyte group it was $50 \%$ in 30 oocytes, with no significant difference between the groups $(p=0.097)$.

The transferrable embryo ratio from the normal morphology oocyte group was $63.3 \%$ and the transferrable embryo in the oval-shaped oocyte group was $46.7 \%$. The Statistical analysis showed no significant difference in embryo quality between the normal oocyte group and the oval-shaped oocyte group ( $p=0.067)$.

\section{DISCUSSION}

Oocyte quality is an important prognostic factor, since the nuclear and cytoplasmic maturity of the oocyte may be directly related to the success rate of ICSI (Ebner et al., 2006; Kahraman et al., 2000). Oocyte and embryo quality were assessed based on morphological features. a dysmorphic oocyte can be regarded as a lower quality oocyte. Dysmorphic oocytes were classified as having a

Table 1. Consensus scoring system for cleavage-stage embryos (in addition to cell number) (ALPHA Scientists In Reproductive Medicine \& ESHRE Special Interest Group Embryology, 2011)

\begin{tabular}{|c|c|c|}
\hline Grade & Rating & Description \\
\hline \multirow{3}{*}{1} & \multirow{3}{*}{ Good } & $<10 \%$ fragmentation \\
\hline & & Stage-specific cell size \\
\hline & & No multinucleation \\
\hline \multirow{3}{*}{2} & \multirow{3}{*}{ Fair } & $10-25 \%$ fragmentation \\
\hline & & $\begin{array}{l}\text { Stage-specific cell size for majority of } \\
\text { cells }\end{array}$ \\
\hline & & No evidence of multinucleation \\
\hline \multirow{3}{*}{3} & \multirow{3}{*}{ Poor } & Severe fragmentation $(.25 \%)$ \\
\hline & & Cell size not stage specific \\
\hline & & Evidence of multinucleation \\
\hline
\end{tabular}




\begin{tabular}{|c|c|c|c|c|c|c|c|c|c|}
\hline \multirow[b]{2}{*}{$\begin{array}{l}\text { Oocyte } \\
\text { morphology }\end{array}$} & \multicolumn{2}{|c|}{ Fertilization rate } & \multirow[b]{2}{*}{$p$ value } & \multicolumn{2}{|c|}{ Cleavage } & \multirow[b]{2}{*}{$p$ value } & \multicolumn{2}{|c|}{ Embryo quality } & \multirow[b]{2}{*}{$p$ value } \\
\hline & Positive & Negative & & Positive & Negative & & $\begin{array}{l}\text { Untrans- } \\
\text { ferrable } \\
\text { embryo }\end{array}$ & $\begin{array}{c}\text { Transferrable } \\
\text { embryo }\end{array}$ & \\
\hline Normal & $\begin{array}{c}41 \\
(68.3 \%) \\
\end{array}$ & $\begin{array}{c}19 \\
(31.7 \%)\end{array}$ & \multirow{2}{*}{$0.444 *$} & $\begin{array}{c}39 \\
(65 \%) \\
\end{array}$ & $\begin{array}{c}21 \\
(35 \%) \\
\end{array}$ & \multirow{2}{*}{ 0.097* } & $\begin{array}{c}22 \\
(36.7 \%) \\
\end{array}$ & $\begin{array}{c}38 \\
(63.3 \%) \\
\end{array}$ & \multirow{2}{*}{$0,067 *$} \\
\hline Oval & $\begin{array}{c}37 \\
(61.7 \%)\end{array}$ & $\begin{array}{c}23 \\
(38.3 \%)\end{array}$ & & $\begin{array}{c}30 \\
(50 \%)\end{array}$ & $\begin{array}{c}30 \\
(50 \%)\end{array}$ & & $\begin{array}{c}32 \\
(53.3 \%)\end{array}$ & $\begin{array}{c}28 \\
(46.7 \%)\end{array}$ & \\
\hline Total & $\begin{array}{c}78 \\
(65 \%)\end{array}$ & $\begin{array}{c}42 \\
(35 \%)\end{array}$ & & $\begin{array}{c}69 \\
(57.5 \%)\end{array}$ & $\begin{array}{c}51 \\
(42.5 \%)\end{array}$ & & $\begin{array}{c}54 \\
(45 \%)\end{array}$ & $\begin{array}{c}66 \\
(55 \%)\end{array}$ & \\
\hline
\end{tabular}

*chi-square

dark cytoplasm, cytoplasmic granularity, cytoplasmic vacuoles, refractive bodies in the cytoplasm, smooth endoplasmic reticulum in the cytoplasm, an oval shape, an abnormal zona pellucida, a large perivitelline space, debris in the perivitelline space, or an abnormal polar body (PB). An oval-shaped oocyte is a dysmorphic oocyte in the IVF cycle. Two possible mechanisms may account for the occurrence of ovoid oocytes: mechanical stress during oocyte puncture and/or the denudation process that deforms the oocyte (Ebner et al., 2008).

Our study reveals that oval oocytes, when compared to normal oocytes, do not yield any difference in fertilization rates, cleavage rates on day 3 and embryo quality. De Sutter et al. (1996) suggested that the morphology of the oocyte bears no relationship to fertilization after ICSI. Balaban et al. (1998) also reported that abnormal oocyte morphology is not associated with a decreased fertilization rate or unfavorable embryo quality. They also stated that embryos derived from abnormal oocytes yield similar clinical pregnancy and implantation rates when transferred, compared with embryos derived from normal oocytes.

Although poor quality oocytes are difficult to fertilize, there is no correlation between ICSI fertilization success and oocyte dimorphism. A failed fertilization in ICSI may occur either because of oocyte activation failure or the oocytes were activated but failed to complete the events leading to fertilization. Another possibility is the unfertilized oocyte containing a decondensed sperm head, indicating failure of oocyte activation, and not a technical error. Savitha et al. (2012) reported that oocyte abnormality can be directly influenced by underlying infertility, effects of ovarian hyperstimulation, and advanced maternal age.

Xia (1997) reported that human oocyte grading can be based on three factors, which are: polar body, size of perivitelline space and cytoplasmic inclusions, and these were significantly related to fertilization rate and embryo quality after ICSI. Loutradis et al. (1999) found that oocyte morphology correlates well with embryo quality and pregnancy rates after ICSI.

Serhal et al. (1997) reported that the outcome of ICSI was dependent on the quality of the oocytes retrieved, they also stated that normal fertilization and early embryo development were achieved in oocytes with abnormal cytoplasm morphology, but the embryos failed to demonstrate the same implantation potential as those derived from oocytes with normal cytoplasm.

Meriano et al. (2001) reported that oocyte dimorphisms were not associated with adverse ICSI outcomes. Khalili et al. (2005) stated that oocyte quality plays a major role in the fertilization process and embryo development in ART programs. Rienzi et al. (2008) reported that morphologic evaluation before ICSI helps to identify MII oocytes with higher developmental potential.
Ebner et al. (2008) stated that Ovoid oocytes with abnormal cleavage pattern show delayed preimplantation development probably due to a reduced number of cell-tocell contacts. Setti et al. (2011) reported that the presence of large polar bodies, large PVS, refractive bodies or vacuoles are associated with decreased oocyte fertilization. They found that the effects of oocyte abnormalities on implantation and pregnancy rates remain unclear.

Braga et al. (2013) stated the individual identification of oocyte dimorphisms might be a prognostic tool for blastocyst development and quality. Yu et al. (2015) reported that the fertilization rate was significantly lower in dysmorphic oocytes than in normal-shaped oocytes, but embryo quality in dysmorphic oocyte group and normal form oocyte group at day 3 was similar.

Fertilization rates, cleavage rates and high quality embryos were not affected by abnormal oocyte morphology but implantation rate was found to be higher in normal morphology oocytes than abnormal morphology oocytes. But we still think that evaluation of MII oocyte quality before ICSI is recommended, leading to higher embryo development.

\section{CONCLUSION}

There is no significant difference in fertilization rates and embryo quality between normal morphology oocytes and oval-shaped oocytes.

\section{ACKNOWLEDGMENTS}

The authors thank all in the team Halim fertility center for their help and support in this study. There is no conflict of interests in this study.

\section{CONFLICT OF INTERESTS}

No conflict of interests has been declared.

\section{Corresponding author:}

Hilma Putri Lubis

HFC IVF center Division of Reproductive

Endocrinology and Infertility Department of Obstetrics and Gynecology

Faculty of Medicine University of Sumatera Utara

Medan, Indonesia

Email: clathrinmine43@yahoo.com

\section{REFERENCES}

Alpha Scientists in Reproductive Medicine and ESHRE Special Interest Group of Embryology. The Istanbul consensus workshop on embryo assessment: proceedings of an expert meeting. Hum Reprod. 2011;26:1270-83. PMID: 21502182 DOI: http://dx.doi.org/10.1093/humrep/der037 
Balaban B, Urman B, Sertac A, Alatas C, Aksoy S, Mercan R. Oocyte morphology does not affect fertilization rate, embryo quality and implantation rate after intracytoplasmic sperm injection. Hum Reprod. 1998;13:3431-3. PMID: 9886529 DOI: http://dx.doi.org/10.1093/humrep/13.12.3431

Braga DP, Setti AS, Figueira Rde C, Machado RB, Iaconelli A Jr, Borges $E$ Jr. Influence of oocyte dysmorphisms on blastocyst formation and quality. Fertil Steril. 2013;100:748-54. PMID: 23768986 DOI: http://dx.doi.org/10.1016/j.fertnstert.2013.05.021

De Sutter P, Dozortsev D, Qian C, Dhont M. Oocyte morphology does not correlate with fertilization rate and embryo quality after intracytoplasmic sperm injection. Hum Reprod. 1996;11:595-7. PMID: 8671274 DOI: http://dx.doi.org/10.1093/HUMREP/11.3.595

Dolgushina NV, Syrkasheva AG, Makarova NP, Kovalskaya EV, Kalinina EA, Sukhikh GT. Correlation between oocyte morphology and the embryo aneuploidy rate in IVF cycles. Gynecol Endocrinol. 2015;31:61-4. DOI: http://dx.doi.org/10.3109/09513590.2015.1086511

Ebner T, Yaman C, Moser M, Sommergruber M, Feichtinger O, Tews G. Prognostic value of first polar body morphology on fertilization rate and embryo quality in intracytoplasmic sperm injection. Hum Reprod. 2000;15:427-30. PMID: 10655316 DOI: http://dx.doi.org/10.1093/humrep/15.2.427

Ebner T, Shebl O, Moser M, Sommergruber M, Tews G. Developmental fate of ovoid oocytes. Hum Reprod. 2008;23:62-6. PMID: 17977865 DOI: http://dx.doi.org/10.1093/humrep/dem280

Ebner T, Moser M, Tews G. Is oocyte morphology prognostic of embryo developmental potential after ICSI? Reprod Biomed Online. 2006;12:507-12. PMID: 16740226 DOI: http://dx.doi.org/10.1016/S1472-6483(10)62006-8

Kahraman S, Yakin K, Dönmez E, Samli H, Bahçe M, Cengiz G, Sertyel S, Samli M, Imirzalioglu N. Relationship between granular cytoplasm of oocytes and pregnancy outcome following intracytoplasmic sperm injection. Hum Reprod. 2000;15:2390-3. PMID: 11056140 DOI: http://dx.doi.org/10.1093/humrep/15.11.2390

Khalili MA, Mojibian M, Sultan AM. Role of oocyte morphology on fertilization and embryo formation in assisted reproductive techniques. Middle East Fertil Soc J. 2005;10:72-7.

Lazzaroni-Tealdi E, Barad DH, Albertini DF, Yu Y, Kushnir VA, Russell $H$, Wu YG, Gleicher N. Oocyte Scoring Enhances Embryo-Scoring in Predicting Pregnancy Chances with IVF Where It Counts Most. Plos One. 2015;10:e0143632. PMID: 26630267 DOI: https://doi.org/10.1371/journal.pone.0143632
Loutradis D, Drakakis P, Kallianidis K, Milingos S, Dendrinos $\mathrm{S}$, Michalas S. Oocyte morphology correlates with embryo quality and pregnancy rate after intracytoplasmic sperm injection. Fertil Steril. 1999;72:240-4. PMID: 10438988 DOI: http://dx.doi.org/10.1016/S0015-0282(99)00233-2

Meriano JS, Alexis J, Visram-Zaver S, Cruz M, Casper RF. Tracking of oocyte dysmorphisms for ICSI patients may prove relevant to the outcome in subsequent patient cycles. Hum Reprod. 2001;16:2118-23. PMID: 11574502 DOI: http://dx.doi.org/10.1093/humrep/16.10.2118 $\begin{array}{lccr}\text { Plachot } \quad \text { M. } & \text { Fertilization. } & \text { Hum } & \text { Reprod. } \\ \text { 2000;15:19-30. } & \text { PMID: } & 11262790 & \text { DOI: } \\ \text { http://dx.doi.org/10.1093/humrep/15.suppl_4.19 } & \end{array}$

Rienzi L, Ubaldi FM, Iacobelli M, Minasi MG, Romano S, Ferrero S, Sapienza F, Baroni E, Litwicka K, Greco E. Significance of metaphase II human oocyte morphology on ICSI outcome. Fertil Steril. 2008;90:1692-700. PMID: 18249393 DOI: http://dx.doi.org/10.1016/j.fertnstert.2007.09.024

Savitha P, Raja DR, Pandiyan R. Normal and Abnormal Oocytes Observed During Assisted Reproductive Technique (ART) Procedures. Chettinad Health City Med J. 2012;1:411.

Serhal PF, Ranieri DM, Kinis A, Marchant S, Davies M, Khadum IM. Oocyte morphology predicts outcome of intracytoplasmic sperm injection. Hum Reprod. 1997;12:1267-70. PMID: 9222015 DOI: http://dx.doi.org/10.1093/humrep/12.6.1267

Setti AS, Figueira RC, Braga DP, Colturato SS, Iaconelli A Jr, Borges E Jr. Relationship between oocyte abnormal morphology and intracytoplasmic sperm injection outcomes: a meta-analysis. Eur J Obstet Gynecol Reprod Biol. 2011;159:364-70. PMID: 21824710 DOI: http://dx.doi.org/10.1016/j.ejogrb.2011.07.031

Xia P. Intracytoplasmic sperm injection: correlation of oocyte grade based on polar body, perivitelline space and cytoplasmic inclusions with fertilization rate and embryo quality. Hum Reprod. 1997;12:1750-5. PMID: 9308806 DOI: http://dx.doi.org/10.1093/humrep/12.8.1750

Yu EJ, Ahn H, Lee JM, Jee BC, Kim SH. Fertilization and embryo quality of mature oocytes with specific morphological abnormalities. Clin Exp Reprod Med. 2015;42:156-62. PMID: 26815385 DOI: http://dx.doi.org/10.5653/cerm.2015.42.4.156 